\title{
Structural studies on M. tuberculosis decaprenyl phosphoryl- $\beta$-D- ribose epimerase-2 enzyme involved in cell wall biogenesis
}

\author{
Shanti P. Gangwar, Arkita Bandyopadhyay and Ajay K. Saxena\#
}

Rm-403/440, Structural Biology Lab, School of Life Sciences, Jawaharlal Nehru University, New Delhi-110067, India.

\# Corresponding author: Ajay K. Saxena, Rm-403/440, Structural biology lab, School of Life Sciences, Jawaharlal Nehru University, New Delhi-110067, India.

Email:ajaysaxena@mail.jnu.ac.in 


\section{Abstract}

The Mycobacterium DprE2 is a NADH-dependent enzyme and converts the decaprenylphosphoryl- $\beta$-D-ribose (DPX) to decaprenylphosphoryl- $\beta$-D-arabinofuranose (DPA). The FAD-containing oxidoreductase MtbDprE1 and NADH-dependent reductase MtbDprE2 enzymes catalyses together the epimerization reaction, which coverts DPR to DPA. Here, MtbDprE2 enzyme was purified and structurally characterized using circular dichroism, molecular modelling and dynamics simulation techniques. The MtbDprE2 was purified, which eluted as oligomer from size exclusion column. The circular dichroism analysis yielded $\sim 47.6 \% \alpha$-helix, $\sim 19.8 \% \beta$-sheet and $\sim 32.6 \%$ random coil structures in MtbDprE2 enzyme and showed highly thermostability. The molecular modelling of $M t b D p r E 2$ and its complex with NADH showed that it contains two domains (i) the large domain consists of central twisted seven $\beta$-sheets decorated by eight $\alpha$-helices and (ii) a small domain contains two short $\alpha$-helices connect by loop. Overall, the MtbDprE2 adopts a typical short-chain dehydrogenase rossmann fold and NADH binds to Asp69, Ser147, Tyr160, Lys164 of catalytic triad and Gly16, Ser19, Glu20, Ile21 of Gly-rich motif of MtbDprE2. 1 ns dynamics simulation was performed on apo and NADH bound MtbDprE2, which indicated the small conformational change in ligand binding site, which resulted more closed pocket than open pocket observed in apo enzyme. Small conformational changes were observed in active site residues and orientation between large and small domains of MtbDprE2 upon NADH binding. Current knowledge of MtbDprE2 structure and its NADH binding mechanism will contribute significantly in development of specific inhibitors against $M$. tuberculosis. 
Keywords: Cell wall biogenesis, MtbDprE2, Circular dichorism, Molecular modeling, Dynamics simulation

Abbreviations used: DprE2, decaprenylphosphoryl- $\beta$-D-ribose epimerase-2. DPR, decaprenylphosphoryl- $\beta$-D- ribofuranose. DPX, decaprenylphosphoryl- $\beta$-D-ribose. DPA, decaprenylphosphoryl- $\beta$-D-arabinofuranose. BTZ043, benzothiazinone. DNB, dinitrobenzamide. E. coli, Escherichia coli. LB, luria Bertani. PME, Partial Mesh Ewald. CD, Circular dichroism. PDB, Protein data bank 


\section{Introduction}

Emergence of multiple drug resistance, extremely drug resistance and total drug resistance strains of M. tuberculosis have affected significantly the treatment of tuberculosis [1]. In

addition, reemergence of infection from dormant stage, slow growth and complex thick cell wall of M. tuberculosis has aggravated the tuberculosis infection. The M. tuberculosis cell wall contains a unique structural components and lipid layers, which makes it impermeable to many drugs and protects the pathogen from host immune system.

The M. tuberculosis DprE1 and DprE2 enzymes are involved in epimerization of DPR to DPA, a precursor required for polysaccharides, arabinogalactan and lipoarabinomannan synthesis in mycobacterial cell wall [2-3]. The DPA covalently attached the mycolic acid to peptidoglycan and involved in complete cell wall synthesis. The MtbDprE2 gene knockout study has shown that it is essential for mycobacterial survival [4] and requires NADH cofactor for its epimerization reaction [5].

The first and second line of tuberculosis drugs inhibit the components of mycobacterial cell wall synthesis [7]. The BTZ043 and DNB are found highly active against multidrug resistant and extensively drug resistant strains of M. tuberculosis. Both series of nitro-aromatic compounds target the heterodimeric decaprenylphosphoryl- $\beta$-D-ribose 20 epimerase enzyme system encoded by MtbDprE1 and MtbDprE2 genes [8, 9]. The BTZ043 has shown low minimal inhibitory concentration $\sim 1 \mathrm{ng} / \mathrm{ml}$ against $M$. tuberculosis, significantly lower than other tuberculosis drugs [8]. The BTZ043 covalently modifies the $M t b D p r E 1$, ablating its function [10] and currently undergoing in clinical trial. Other inhibitors of MtbDprE1 (i) 2-mercaptobenzothiazole [11] (ii) 1, 2, 3 triazole conjugates [12] (iii) next generation banzothiazinones [13] and (iv) non-covalent inhibitors from scaffold 
morphing [14] have been identified and characterized. The crystal structures of several complexes of $M t b D p r E 1$ with synthetic inhibitors have been also determined $[\mathbf{1 5}, \mathbf{1 6}]$.

The MtbDprE1 and MtbDprE2 proteins are essential for mycobacterial growth [17] and interacts to each other and considered as two subunit of single enzyme, decaprenyl phosphoryl- $\beta$-D-ribofuranose 2-epimerase. In Corynebacterium glutamicium, the bacterial two hybrid system has shown the interaction between DprE1 and DprE2 enzymes [18]. The MtbDprE1 and MtbDprE2 complex model has been proposed using molecular modeling, threading and dynamics simulation techniques [19]. The mutational and dynamics simulation analysis on InhA-NADH complex has shown the mechanism of drug resistance in M. tuberculosis [20].

So far, little is known about structure and mechanism of MtbDrprE2 enzyme. The analysis of structure and NADH binding of MtbDprE2 will be critical in development of specific inhibitors against M. tuberculosis. In present study, the MtbDprE2 was purified and secondary structure analysis was performed using circular dichroism spectroscopy. Molecular modeling and dynamics simulation analysis on wild type MtbDprE2 and its complex with NADH were performed to understand the structural basis of NADH binding to MtbDprE2.

\section{Materials and methods}

\subsection{Expression and purification}

$M t b D p r E 2$ gene $[R v 3791,254$ residues, $27 \mathrm{kD}]$ was cloned in $p E T 28 a(+)$ expression vector using NdeI and XhoI restriction sites. Forward (5'- GATCCATAT GATGGTTCTTGATGC CGTA -3') and reverse (5'- CATGCTCGAGTCAGATGGGCAGCTTGCG- 3') primers were used for MtbDprE2 gene amplification from $H 37 R v$ genomic DNA. The resulting 
$M t b D p r E 2$ plasmid was checked by restriction-digestion experiment. The MtbDprE2 plasmid contains $6 \mathrm{xHis}$ tag and thrombin cleavage site at N-terminal and 254 residues of MtbDprE2. The MtbDprE2 plasmid was transformation in Escherichia coli BL21(DE3) cells, however protein expressed as inclusion body. Different variables, IPTG concentration, temperature, various $E$. coli cell lines were tried, however protein expressed only as inclusion body in cell.

The MtbDprE2 gene was further cloned in $p E T-S U M O$ vector (Invitrogen) using TA cloning method and checked by gene sequencing. The E. coli BL21(DE3) cells were used for $M t b D p r E 2$ plasmid transformation. The $31 \mathrm{LB}$ media $(50 \mu \mathrm{g} / \mathrm{ml} \mathrm{Kanamycin} \mathrm{as}$ antibiotics) was to grow single colony and grown at $37^{\circ} \mathrm{C}$, till $\mathrm{OD}_{600} \sim 0.7-0.8 \cdot 0.25 \mathrm{mM}$ IPTG was used to induce the culture at $37^{\circ} \mathrm{C}$ and grew further for $3 \mathrm{hr}$. The culture was centrifuged at 10,000 x g, collected the pellet and suspended in buffer-A (20 mM Tris/HCl pH 8.0, 5\% (v/v) Glycerol, $300 \mathrm{mM} \mathrm{NaCl}, 1 \mathrm{mM}$ Benzamidine-HCl, $1 \mathrm{mM}$ Phenylmethylsulfonyl fluoride, $2 \mathrm{mM} \beta$-mercaptoethanol and $0.3 \mathrm{mg} / \mathrm{ml}$ Lysozyme). The culture was kept on ice for $1 \mathrm{~h}$, sonicated and supernatant was collected by centrifugation at $18,000 \mathrm{x} g$ for $20 \mathrm{~min}$.

The Ni-NTA column was pre-equilibrated with buffer-B $(20 \mathrm{mM}$ Tris/ $\mathrm{HCl} \mathrm{pH} 8.0$, $300 \mathrm{mM} \mathrm{NaCl}$, 5\% Glycerol, $1 \mathrm{mM}$ Phenylmethylsulfonyl fluoride, $1 \mathrm{mM}$ Benzamidine$\mathrm{HCl}$ and $2 \mathrm{mM} \beta$-mercaptoethanol) and loaded the supernatant. The column was washed with buffer-B $+15 \mathrm{mM}$ Imidazole and eluted the protein in buffer- $\mathrm{B}+300 \mathrm{mM}$ imidazole. The peak fractions were pooled, concentrated and loaded on Superdex 75(16/60) column (GE Healthcare) preequilibrated in buffer $20 \mathrm{mM}$ Tris/ $\mathrm{HCl} \mathrm{pH} 8.0,300 \mathrm{mM} \mathrm{NaCl}$ and $5 \%$ 
Glycerol. We pooled the peak fractions and used ultracentrifugal device (Amicon) to concentrate the protein.

The SUMO protease (a ubiquitin like protein processing enzyme) was used to cleave SUMO tag from MtbDprE2-SUMO fusion protein. $200 \mu \mathrm{g}$ of $M t b D p r E 2-S U M O$ fusion protein was dissolved in $500 \mu \mathrm{l}$ of SUMO protease in buffer $(500 \mathrm{mM}$ Tris/ $\mathrm{HCl} \mathrm{pH} 8.0,1.5$ $\mathrm{M} \mathrm{NaCl}, 2 \% \mathrm{NP}-40,10 \mathrm{mM}$ DTT) and $2 \mu \mathrm{g}$ of SUMO protease was added in it. The reaction mixture was incubated at $4{ }^{\circ} \mathrm{C}$ and aliquots were taken out after $4,8,16$ and $24 \mathrm{~h}$. The aliquots were examined on SDS-PAGE to check the cleavage reaction. The cleaved MtbDprE2 was further purified using Superdex 75(16/60) column. The purity of MtbDprE2 was analyzed on SDS-PAGE and estimated the concentration using Bradford method.

\subsection{Circular dichorism analysis}

Chirascan ${ }^{\mathrm{TM}} \mathrm{CD}$ spectropolarimeter (Applied Photophysics) was used to collect the CD data on MtbDprE2 in 260-200 nm wavelength. The MtbDprE2 was concentrated to $0.1 \mathrm{mg} / \mathrm{ml}$ in b10mM Tris/HCl buffer $\mathrm{pH} 8.0$ buffer and loaded on 1-mm sample cuvette for $\mathrm{CD}$ data collection. The protein buffer was used as blank and subtracted from each reading. Three sequential scans were collected for each data. The mean residue ellipticity (deg. $\left.\mathrm{cm}^{2} / \mathrm{dmol}\right)$ was calculate using Dichroweb server [21]. For thermal denaturation analysis, the CD data on $M t b D p r E 2$ was collected from $20^{\circ} \mathrm{C}$ to $70^{\circ} \mathrm{C}$ in $10^{\circ} \mathrm{C}$ interval. Before measurement, the sample cuvette was incubated at each temperature and checked with water bath.

Various theoretical structural prediction programs e. g. Jpred [22], Raptor X [23], HNN [24], DSC [25], PHD [26], GOR [27], CFSSP [28] and SOPMA [29] were used to calculate the secondary structure of $M t b D p r E 2$. The PSIPRED [30] analysis on $M t b D p r E 2$ 
sequence is shown in Fig. 2A.

\subsection{Homology modeling}

The ITASSER server [31] was used to obtain the MtbDprE2 model (1-254 residues).

LOMETS software [32] used the MtbDprE2 sequence and identified the best template from protein database. The SPICKER program [33] identified the best template with high Z-score (high threading alignment score) after simulation of structure assembly. Based on the Cscore, the program predicts the best score. The TM-align program yielded the top 10 models having best TM-score. PROCHECK [34] program was used analyzed the quality of MtbDprE2 model.

\subsection{MtbDprE2-NADH docking analysis}

The PDB-4JRO [Crystal structure of FabG+NADP+ complex from Listeria monocytogenes] was used as template to dock NADH into MtbDprE2 model. To optimize NADH docking, the GLIDE program [35] of Schrödinger-9.4 suite was used with IFD (induced fit docking) module. We used the XP (Extra Precision) scoring function and scaled the van der Waals radii of $M t b H d d A$ by 0.6 fold. For prime site optimization, all MtbDprE2 residues located within $4 \AA$ radii of NADH were refined. The Glidestone module of Glide program was used to obtain the best MtbDprE2+NADH complex using 5,000 cycles of scoring and 5,000 cycles of minimization. The MtbDprE2+NADH complex having lowest IFD value was selected as the best $M t b D p r E 2+\mathrm{NADH}$ complex.

\subsection{Dynamics simulation analysis}


The dynamics simulation on apo-MtbDprE2 and its complex with NADH were performed using GROMACS 2020.1-MODIFIED version [36] taking OPLSAA force field [37]. The protein was kept in cubic box having $0.6 \mathrm{~nm}$ spacing and filled with TIP3P water molecules. The entire charge of system was neutralized by adding chloride and sodium atoms and periodic boundary condition was used for dynamics simulation. The system was subjected to 5000 steps of steepest descent minimization. Before dynamics, the solvent and ions were equilibrated surrounding the protein. Initially, first phase of NVT equilibration (100 ps) was performed, which stabilize the temperature of the system. In second phase, equilibration of pressure was conducted in 100 ps NPT equilibration (constant number of particles, pressure and temperature).

A constant temperature $300 \mathrm{~K}$ and coupling constant $(\tau) \sim 0.1$ ps was maintained by applying V-rescale (modified Berendsen thermostat) coupling with coulomb cutoff 1 . The 1 atm pressure was maintained, isotropic scaling and 2 ps relaxation time. LINCS algorithm was used to constrain the bond lengths. The long ranger electrostatic interactions was calculated using PME method [38]. A force constant of $1000 \mathrm{~kJ} / \mathrm{mol} . \mathrm{nm}^{2}$ was used to restrain the protein harmonically. 1 ns molecular dynamics simulation with time step $\sim 2$ fs was performed and saved the coordinates after every 5 ps for trajectory analysis. The least square fitting procedure was used to calculate RMSD and RMSF and plotted by Plot2X program [39]. PROCHECK program [34] was used to check the stereochemistry of simulated MtbDprE2 models and Chimera program [40] for structure visualization.

\section{Results and discussion}

\subsection{Purified MtbDprE2 exists as oligomer}


MtbDprE2 sequence (254 residues, $27 \mathrm{kDa}$ ) is shown in Fig. 1A and residues involved in NADH binding are shown in blue letters. The MtbDprE2 gene was cloned into $p E T 28(\mathrm{a}+)$ expression vector, however protein expressed as inclusion body in E. coli. BL21(DE3) cells. Various parameters e. g. IPTG concentrations $(0.1-1 \mathrm{mM})$, different temperature (10 $37{ }^{\circ} \mathrm{C}$ ) and different cells (E. coli arctic, BL21-codon-Plus) were tried, however protein expressed insoluble fraction of cell. The MtbDprE2 gene was further cloned in $p E T-S U M O$ vector using TA cloning method and clones were confirmed restriction-digestion (Fig. 1B). The E. coli. BL21(DE3) cells were used for MtbDprE2 plasmid transformation and protein overexpressed as soluble protein. The MtbDprE2 fusion protein eluted as oligomer from Superdex 200(16/60) column. The MtbDprE2 fusion protein was treated with SUMO protease and examined on SDS-PAGE (Fig. 1C, inset). The SDS-PAGE analysis showed the fusion protein, cleaved DprE2 and cleaved SUMO tag (Fig. 1C, inset). The cleaved MtbDprE2 was further purified on Superdex 200(16/60) column, which eluted as oligomer from column (Fig. 1C).

\subsection{Secondary structure and thermal denaturation analysis}

The PSIPred analysis [30] on MtbDprE2 sequence (Fig. 2A) showed seven $\beta$-strands and ten $\alpha$-helices structures in protein. The CD data was collected in 260-200 nm wavelength range and secondary structure was calculated using DICHROWEB server [41]. The program yielded $\sim 47.6 \% \alpha$-helix, $\sim 19.8 \% \beta$-sheet and $\sim 32.1 \%$ random coil in MtbDprE2 structure (Fig. 2B). Theoretical structure prediction on MtbDprE2 also yielded quite similar secondary structure, as observed in CD data (Table 1). For thermal denaturation analysis, $\mathrm{CD}$ data on MtbDprE2 was collected from $20{ }^{\circ} \mathrm{C}-70{ }^{\circ} \mathrm{C}$ in $10{ }^{\circ} \mathrm{C}$ step (Fig. 2C). These data 
showed that minor changes in secondary structures, indicating high thermostability of MtbDprE2 enzyme.

\subsection{Molecular modeling}

The I-TASSER server was used to obtain the MtbDprE2 model (1-254 residues). The server identified the PDB-4JRO (Crystal structure of FabG + NADP+ complex from Listeria monocytogenes) as the best template with following parameters $e . g$. $(\mathrm{id} 1=0.20, \mathrm{id} 2=0.24$, $\operatorname{cov}=0.93$, Z-score $=2.07)$. The best MtbDprE2 model was obtained with following parameters $[\mathrm{C}$-score $=-0.74$ and TM-score $=0.62 \pm 0.14]$. The PROCHECK program showed the good stereochemistry of obtained MtbDprE2 model and all residues lie in allowed $\phi, \psi$ regions of Ramachandran plot (Fig. S1). The ProSA [42] and ERRAT [43] servers analysis on $M t b D p r E 2$ model also showed the good stereochemistry and high reliability (Fig. S1).

The MtbDprE2 model belongs to short chain dehydrogenase/ reductase (SDR) superfamily of enzymes with Rossman fold containing NADH/NAD(P)H binding site. The MtbDprE2 model contains two domains, (i) the major domain, which adopts a typical rossman-fold with seven stranded $\beta$-sheets surrounded by eight- $\alpha$ helices and (ii) a small domain contains two $\alpha$-helices (a6-a7) connected by a loop (Fig. 3A). The electrostatic surface diagram of MtbDprE2 contoured at $\pm 10 \mathrm{kT}$ (Fig. 3B) showed that NADH binding site contains partially positively charge, while overall surface of enzyme is more negatively charge and partial positive charges are scattered all over the surface.

\subsection{NADH binding analysis}


The PDB-4JRO was used as template to dock NADH into MtbDprE2. Obtained MtbDprE2+NADH complex was used as template in GLIDE program of schrÖdinger suite to optimize the fitting of NADH into MtbDprE2 and induced fit docking protocol was used for it.

The LigPlot [44] analysis showed that NADH occupies the deep cleft of MtbDprE2 and forms hydrogen bonds with Asp69, Ser147, Tyr160, Lys164 residues of catalytic triad, Gly16, Ser19, Glu20, Ile21 residues of Gly-rich motif and Leu42, Ala95 residues of MtbDprE2 (Fig. 3C). In SDR family members, the active site is usually formed by Tyr-X-XX-Lys sequence [45, 46]. In MtbDprE2, Tyr160-Gly161-Ser162-Thr163-Lys164 motif is found in catalytic site, as observed in 'classical' type of SDR superfamily. The Asn99 residue of MtbDprE2 is highly conserved to SDR family of proteins, however, does not interact to NAD in MtbDprE2. Instead, highly conserved Asp69 interacts with NAD and forms the catalytic triad. Lys 164 forms hydrogen bond with NAD nicotinamide ribose and decreased the $\mathrm{pKa}$ of Tyr160-OH. The Tyr160 catalyzes and Ser147 immobilizes the substrate and thus forms the Ser147, Tyr160, Lys164 catalytic triad.

The TG-xxxGxG consensus sequence in SDR family enzymes is involved in NADH binding [47]. In MtbDprE2, the Gly rich motif L15-G16-G17-T18-S19-E20-I21-G22-L23A24 (shown in \#) is observed at first $\beta 1$ - strand. The Gly16 residue of MtbDprE2 is highly conserved, while S19, E20 and I21 residues are partially conserved and interacts with 2'phosphate and ribose hydroxyl groups of NADH.

The $\beta 4-\alpha 4$ loop of $M t b D p r E 2$ contains ${ }^{91}$ NNAGX ${ }^{95}$ motif, which stabilizes the central $\alpha 6$ helix and $\beta$-sheet, which contains two catalytic residues (Tyr157 and Lys161) $[48,49]$. In MtbDprE2, ${ }^{90}$ DVAIVAFGL ${ }^{99}$ motif represents the same loop though conserved, 
but may play a role like ${ }^{91} \mathrm{NNAGX}{ }^{95}$ motif, as found in other short chain dehydrogenase enzyme.

In apo-MtbDrpE2, the NADH binding pocket is quite wide (Fig. 3B), compared to ligand bound, as surrounding helices tilted outwards from the pocket binding (Fig. 3E). The MtbDprE2 showed little conservation and similarity with other members of SDR family of enzymes and pocket involved in substrate binding has shown high degree of variability [50].

\subsection{Sequence alignment and comparative structure analysis}

The MtbDprE2 structure was aligned with structures of PDB database using TM-structural alignment program of I-TASSER server, which yielded ten closest structural homologs (as shown in Table 2), (i) PDB-3awd, identity=17.4\%, 97.2\% coverage [51] (ii) PDB-3s55, identity $=18.4 \%, 96.5 \%$ coverage [52] (iii) PDB-1gee, identity $=15.4 \%, 96.9 \%$ coverage (iv) PDB-2uvd, identity $=15.6 \%, 95.7 \%$ coverage $[53](v)$ PDB-3aut, identity $=16.6 \%$, coverage $=97.2 \%[54]$ (vi) PDB-3t7c, identity $=13.3 \%$, coverage $=97.2 \%$ [55] (vii) PDB-1ipe, identity $=18.1 \%$, coverage $=97.6 \%$ [56] (viii) PDB-2wsb, identity $=18.4 \%$, coverage $=96.5 \%$ [57] (ix) $\mathrm{PDB}=2 \mathrm{uve}$, identity=15\%, coverage=97.2\% [58] (x) PDB-4nbv, identity=21\%, Coverage $=95.7 \%[59]$.

Sequence alignment of MtbDprE2 with ten structural homologs (Fig. 4A) showed that Asp69, Ser147, Tyr160, Lys164 of catalytic triad (shown as *) are highly conserved in all ten sequences. In addition, Gly16, Ser19, Glu20, Ile21 of Gly-rich motif of MtbDprE2 are also fully and partially conserved. Two additional residues, Leu44, Ala95 (shown as ${ }^{+}$) are least conserved in ten sequences. Overall, residues of NADH binding pocket of MtbDprE2 were quite conserved in all ten homologs, despite having low sequence identity 
(13-21\%) (Table 3). Superposition of structures of ten homologs on MtbDprE2 has yielded $\mathrm{RMSD} \sim 0.91$ for $184 \mathrm{Ca}$ atoms, indicating quite conserved structures in all sequences (Fig. 4B). Major differences were observed at N- and C-terminal regions, small domain comprising $\alpha 6-\alpha 7$ helices and in a small loop (shown in circle) in MtbDprE2 and ten homologs, while remaining structure was quite conserved.

\subsection{Dynamics simulation on wild type MtbDprE2 and its complex with NADH}

To examine the MtbDprE2 enzyme dynamics involved in NADH binding, we performed dynamics simulation on apo and NADH bound MtbDprE2 and analyzed the trajectories obtained during simulation.

\section{Dynamics simulation on apo-MtbDprE2}

$1 \mathrm{~ns}$ dynamics simulation was performed on apo-MtbDprE2 and analyzed the conformational changes occurred during simulation. The simulated MtbDrprE2 structure (red) was superposed on starting structure (grey), which yielded RMSD $\sim 2.5 \AA$ for $240 \mathrm{C} \alpha$ atoms (Fig. 5A). Overall MtbDprE2 structure superposed well, except minor changes were observed in loop regions and helical orientation. Major changes in RMSD occurred during $0.1 \mathrm{~ns}$ simulation and remained stable to $\sim 0.25 \mathrm{~nm}$ during $1 \mathrm{~ns}$ simulation (Fig. 5D). Radius of gyration, $\operatorname{Rg} \sim 1.82 \pm 0.20 \mathrm{~nm}$ was observed during $1 \mathrm{~ns}$ simulation (Fig. 5E). To examine, the structural changes in $M t b D p r E 2$, we computed the RMSF of MtbDprE2 residues (Fig. 5F). High RMSF were obtained for N- and C-terminal regions, while remaining structure showed RMSF between 0.1-0.2 nm. The Asp69, Ser147, Tyr160, Lys164 of catalytic triad 
and Gly16, Ser19, Glu20, Ile21 of Gly-rich motif, involved in NADH binding were quite stable during dynamics simulation.

\section{Dynamics simulation with MtbDprE2+NADH complex}

1 ns dynamics simulation was performed on $M t b D p r E 2+\mathrm{NADH}$ complex to analyze the enzyme dynamics involved in NADH binding. The simulated complex structure (cyan) was superposed on starting structure (grey), which yielded RMSD $\sim 2.49 \AA$ for $224 \mathrm{C} \alpha$ atoms (Fig. 5B). The NADH binding inducing small conformational change in NADH binding pocket, which result in more closed pocket than open pocket observed in wild type MtbDprE2. The orientation between large and small domains of MtbDprE2 domains also changes slightly after dynamics simulation (Fig. 5B).

Major changes in RMSD occurred during $0.2 \mathrm{~ns}$ simulation and then remained stable to $\sim 0.35 \mathrm{~nm}$ throughout $1 \mathrm{~ns}$ simulation. The radius of gyration, $\mathrm{Rg} \sim 1.78 \pm 0.3 \mathrm{~nm}$ was observed during entire simulation (Fig. 5E). To examine the structural changes, we computed the RMSF for each residue of MtbDprE2 (Fig. 5F). High RMSF values were observed for $\mathrm{N}$ - and C-terminal regions, 110-120 residues, 180-190 residues and 200-225 residues of $M t b D p r E 2$. Residues involved in NADH binding did show high RMSF values in dynamics simulation of MtbDprE2+NADH complex.

\section{Conclusion}

The MtbDprE2 enzyme is involved in DPA biosynthetic pathway and critical for $M$. tuberculosis drug development. Here, we have purified and structurally characterized the NADH binding mechanism of MtbDprE2. The CD analysis showed the secondary 
structures of $M t b D p r E 2$, quite similar to SDR family of enzymes. The thermal denaturation profile of MtbDprE2 indicated the high thermostability of enzyme. We have modelled and performed dynamics simulation on apo and NADH bound MtbDprE2 to understand the enzyme dynamics involved in NADH recognition. The NADH binding to MtbDprE2 showed minor conformational changes in active site residues and orientation between and small and large domains in dynamics simulation. The knowledge of structure and dynamical aspect of NADH binding may contribute in specific inhibitors development of $M t b D r E 2$ enzyme.

\section{Conflict of interest statement}

None

\section{Acknowledgements}

The partial funding from DST-PURSE, UPE-II, UGC resource networking and Department of Biotechnology (No. PAC-SLS-AKS-DBT-01131216-737) are gratefully acknowledged. JNU, AIRF facility for their kind support in conducting the CD experiment. ShantiPal and Arkita were supported by fellowship from UGC. 


\section{References}

1. Udwadia, Z. F.; MDR, XDR, TDR tuberculosis: ominous progression. Thorax., 2012, 67, 286-288.

2. Mikusova, K.; Huang, H.; Yagi, T.; Holsters, M.; Vereecke, D.; D'Haeze, W., et al. Decaprenylphosphoryl arabinofuranose, the donor of the D-arabinofuranosyl residues of mycobacterial arabinan, is formed via two-step epimerization of decaprenylphosphoryl ribose. J. Bacte., 2005, 187, 8020-8025.

3. Wolucka, B. A. Biosynthesis of D-arabinose in mycobacteria - a novel bacterial pathway with implications for antimycobacterial therapy. The FEBS journal, 2008, 275, 26912711.

4. Sassetti, C. M.; Boyd, D. H.; Rubin, E. J. Genes required for mycobacterial growth defined by high density mutagenesis. Mol. Micro., 2003, 48, 77-84.

5. Trefzer, C.; Rengifo-Gonzalez, M.; Hinner, M. J.; Schneider, P.; Makarov, V.; Cole, S. T. et al. Benzothiazinones: prodrugs that covalently modify the decaprenylphosphorylbeta-D-ribose2'-epimerase DprE1 of Mycobacterium tuberculosis. Jour. Amer. Chem. Soc., 2010, 132, 13663-13665.

6. Manina, G.; Pasca, M. R.; Buroni, S.; De Rossi, E.; Riccardi, G. Decaprenylphosphorylbeta-D-ribose 2'-epimerase from Mycobacterium tuberculosis is a magic drug target. Curr. Med. Chem., 2010, 17, 3099-3108.

7. Hett, E. C.; Rubin, E. J. Bacterial Growth and Cell Division: a Mycobacterial Perspective. Microbiol Mol Biol Rev., 2008, 72(1), 126-156.

8. Makarov, V.; Manina, G.; Mikusova, K.; Mollmann, U.; Ryabova, O.; Saint-Joanis, B. et al. Benzothiazinones kill Mycobacterium tuberculosis by blocking arabinan synthesis. Science, 2009, 324, 801-804.

9. Christophe, T.; Jackson, M.; Jeon, H.K.; Fenistein, D.; Contreras-Dominguez, M.; Kim, J. et al. High content screening identifies decaprenyl-phosphoribose 2' epimerase as a target for intracellular antimycobacterial inhibitors. PLoS patho., 2009, 5, e1000645.

10. Trefzer, C.; Skovierova, H.; Buroni, S.; Bobovska, A.; Nenci, S.; Molteni, E. et al. Benzothiazinones are suicide inhibitors of mycobacterial decaprenylphosphoryl-beta-Dribofuranose 2'-oxidase DprE1. J. Am. Chem. Soc., 2012, 134, 912-915.

11. Mir, F.; Shafi, S.; Zaman, M. S.; Kalia, N. P.; Rajput, V. S.; Mulakayala, C.; Mulakayala, N.; Khan, I.A.; Alam, M.S. Sulfur rich 2-mercaptobenzothiazole and 1,2,3triazole conjugates as novel antitubercular agents. Eur. J. Med. Chem., 2014, 76, 274283. 
12. Boechat, N.; Ferreira, V.F.; Ferreira, S.B.; Ferreira, M.L.G.; da Silva, F.C.; Bastos, M.M.; Costa, M.S.; Lourenço, M.C.S.; Pinto, A.C.; Krettli, A.U.; Aguiar, A.C.; Teixeira, B.M.; da Silva, N.V.; Martins, P.R.C.; Bezerra, FAFM; Camilo, ALS; da Silva, GP; Costa CCP. Novel 1,2,3-triazole derivatives for use against Mycobacterium tuberculosis H37Rv (ATCC 27294) strain. J. Med. Chem., 2011, 54, 5988-5999.

13. Makarov, V.; Lechartier, B.; Zhang, M.; Neres, J.; van der Sar, A.; Raadsen, S.; Hartkoorn, R.; Ryabova, O.; Vocat, A.; Decosterd, L.; Widmer, N.; Buclin, T.; Bitter, W.; Andries, K.; Pojer, F.; Dyson, P.; Cole, S. Towards a new combination therapy for tuberculosis with next generation benzothiazinones. EMBO Mol. Med., 2014, 6, 372383.

14. Shirude, P. S. et al. Azaindoles: noncovalent DprE1 inhibitors from scaffold morphing efforts, kill Mycobacterium tuberculosis and are efficacious in vivo. J. Med. Chem., 2013, 56, 9701-9708

15. F. Wang, D.; Sambandan, R.; Halder, J.; Wang, S.; Batt, B.; Weinrick, I.; Ahmad, P.; Yang, Y.; Zhang, J.; Kim, M.; Hassani, S.; Huszar, C.; Trefzer, T.; Kaneko, K.; Mdlill, S.; Franzblau, A.; Chatterjee, K.; Johnsson, K.; Milkusova, G.; Besra, K.; Futterer, S.; Robbins, W.; Bames, P. Schultz. Identification of a small molecule with activity against drug-resistant and persistent tuberculosis. Proc. Nat. Acad. Sci., 2013, 27, E2510-2517.

16. Batt, S.; Jabeen, T.; V. Bhowruth, L.; Quill, P.; Lund, L.; Eggeling, L.; Alderwick, K.; Futterer, G. Besra. Structural basis of inhibition of Mycobacterium tuberculosis DprE1 by benzothiazinone inhibitors. Proc. Nat. Acad. Sci., 2012, 109, 11354-11359.

17. Kolly, G. S.; Boldrin, F.; Sala, C.; Dhar, N.; Hartkoorn, R.C.; Ventura, M.; Serafini, A.; McKinney, J.D.; Manganelli, R.; Cole, S.T. Assessing the essentiality of the decaprenylphospho-d-arabinofuranose pathway in Mycobacterium tuberculosis using conditional mutants. Mol. Microbiol., 2014, 92, 194-211.

18. Jankute, M.; Byng, C.V.; Alderwick, L.J.; Besra, G.S. Elucidation of a protein-protein interaction network involved in Corynebacterium glutamicum cell wall biosynthesis as determined by bacterial two-hybrid analysis. Glycoconj. J., 2014, 31, 475-483.

19. Bhutani, I.; Loharch, S.; Gupta, P.; Madathil, R.; Parkesh, R. Structure, dynamics, and interaction of Mycobacterium tuberculosis (Mtb) DprE1 and DprE2 examined by molecular modeling, simulation, and electrostatic studies. PLoS ONE, 2015, 10, e0119771.

20. Schroeder, E. K.; Basso, L. A.; Santos, D. S.; de Souza, O. N. Molecular dynamics simulation studies of the wild-type, I21V, and I16T mutants of isoniazid-resistant Mycobacterium tuberculosis enoyl reductase (InhA) in complex with NADH: toward the understanding of NADH-InhA different affinities. Biophy. J., 2005, 89, 876-884. 
21. Whitmore, L.; Wallace, B. A. Protein secondary structure analyses from circular dichroism spectroscopy: methods and reference databases. Biopolymers., 2008, 89, 392-400.

22. Cole, C.; Barber, J. D.; Barton, G. J. The Jpred 3 secondary structure prediction server. Nucl. Acid. Res., 2008, 36, W197-W201.

23. Wang, S.; Li, W.; Liu, S.; Xu, J. RaptorX-Property: a web server for protein structure property prediction. Nucl. Acid. Res., 2016, 44, W430-W435.

24. Lin, K.; Simossis, V. A.; Taylor, W. R.; Heringa, J. HNN-A simple and fast secondary structure prediction method using hidden neural networks. Bioinformatics, 2004, 21, 152-159.

25. King, R. D.; Saqi, M. A. S.; Sayle, R. A.; Sternberg, M. J. E. DSC: public domain protein secondary structure predication. Computer applications in the biosciences, 1997, 13, 473-474.

26. Rost, B.; Sander, C.; Schneider, R. PHD-an automatic mail server for protein secondary structure prediction. Bioinformatics, 1994, 10, 53-60.

27. Kouza, M.; Faraggi, E.; Kolinski, A.; Kloczkowski, A. The GOR method of protein secondary structure prediction and its application as a protein aggregation prediction tool. Methods Mol Biol., 2017, 1484, 7-24.

28. Kumar, T. A. CFSSP: Chou and Fasman secondary structure prediction server. Wide Spectrum, 2013, 1, 15-19.

29. Geourjon, C.; Deleage, G. SOPMA: significant improvements in protein secondary structure prediction by consensus prediction from multiple alignments. Bioinformatics, 1995, 11, 681-684.

30. Jones, D. T. Protein secondary structure prediction based on position-specific scoring matrices. J. Mol. Biol., 1999, 292, 195-202.

31. Zhang, Y. I-TASSER server for protein 3D structure prediction. BMC bioinformatics, 2008, $9(1), 9-40$.

32. Wu, S.Y.; Zhang,Y. LOMETS: a local meta-threading-server for protein structure prediction. Nuc. Aci. Res. 2007, 35, 3375-3382.

33. Zhang, Y.; Skolnick, J.; SPICKER: A Clustering Approach to Identify Near-Native Protein Folds. J. Compu. Chem. 2004, 25, 865-871.

34. R. A. Laskowski, M. W. MacArthur, D. S. Moss, J. M. Thornton, PROCHECK: a program to check the stereochemical quality of protein structures. J. Appl. Cryst., 1993, 26, 283-291. 
35. Friesner, R. A.; Murphy, R. B.; Repasky, M. P.; Frye, L. L.; Greenwood, J. R.; Halgren, T. A.; Sanschagrin, P. C.; Mainz, D. T. Extra Precision Glide: Docking and Scoring Incorporating a Model of Hydrophobic Enclosure for Protein-Ligand Complexes. J. Med. Chem., 2006, 49, 6177-6196.

36. Hess, B.; Kutzner, C.; Van Der Spoel, D.; Lindahl, E. GROMACS 4: algorithms for highly efficient, load-balanced, and scalable molecular simulation. J. Chem. The. Сотри., 2008, 4, 435-447.

37. Oostenbrink, C.; Villa, A.; van Gunsteren, W. F. A biomolecular force field based on the free enthalpy of hydration and solvation: the GROMOS force-field parameter sets 53A5 and 53A6. J. Comput. Chem., 2004. 25, 1656-1676.

38. Harvey, M.; De Fabritiis, G. An implementation of the smooth particle mesh Ewald method on GPU hardware. J. Chem. Theo. Comp., 2009, 5, 2371-2377.

39. Kalesinskas, L.; Cudone, E.; Fofano, Y.; Putonti, C. S-plot2: Rapid Visual and Statistical Analysis of Genomic Sequences. Evolutionary. Bioinformatics, 2018, 14, 11769343-18797354.

40. Pettersen, E. F. ; Goddard, T. D.; Huang, C.C.; Couch, G. S.; Greenblatt, D. M.; Meng, E. C.; Ferrin, T. E. UCSF chimera-a visualization system for exploratory research and analysis. J. Comput. Chem. 2004, 25(13), 1605-1612.

41. Whitmore, L.; Wallace, B. DICHROWEB, an online server for protein secondary structure analyses from circular dichroism spectroscopic data. Nucl. Acid. Res. 2004, 32, W668-W673.

42. Wiederstein, M.; Sippl, M. J. ProSA-web: interactive web service for the recognition of errors in three-dimensional structures of proteins. Nucl. Acid. Res., 2007, 35, W407-W410.

43. Colovos, C.; Yeates, T. O. ERRAT Verification of protein structures: patterns of nonbonded atomic interactions. Protein Sci., 1993, 2(9), 1511-1519.

44. Wallace, A. C.; Laskowski, R. A.; Thornton, J. M. LIGPLOT: a program to generate schematic diagrams of protein-ligand interactions. Protein Eng., 1996, 8, 127-134.

45. Jornvall, H.; Persson, B.; Krook, M.; Atrian, S.; Gonzalez-Duarte, R.; Jeffery, J., et al. Short-chain dehydrogenases/reductases (SDR). Biochemistry, 1995, 34, 6003-6013.

46. Filling, C.; Berndt, K.D.; Benach, J.; Knapp, S.; Prozorovski, T.; Nordling, E., et al. Critical residues for structure and catalysis in short-chain dehydrogenases/reductases. J. Bio. Chem., 2002, 277, 25677-25684.

47. Persson, B.; Kallberg, Y.; Oppermann, U.; Jornvall, H. Coenzyme-based functional assignments of short-chain dehydrogenases/reductases (SDRs). Chemico-biological interactions, 2003, 143-144, 271-278. 
48. Oppermann, U.; Filling, C.; Hult, M.; Shafqat, N.; Wu, X.; Lindh, M., et al. Short-chain dehydrogenases/reductases (SDR): the 2002 update. Chemico-biological interactions. 2003, 143-144, 247-53.

49. Kavanagh, K.; Jörnvall, H.; Persson, B.; Oppermann, U. Medium and short chain dehydrohenase /reductase gene and protein families: The SDR superfamily :functional and structural diversity within a family of metabolic and regulatory enzymes. Cell Mol. Life Sci., 2008, 65, 3895-3906.

50. Jornvall, H.; Persson, B.; Krook, M.; Atrian, S.; Gonzalez-Duarte, R.; Jeffery, J. et al. Short-chain dehydrogenases/reductases (SDR). Biochemistry, 1995, 34, 6003-6013.

51. Liu, X.; Yuan, Z.; Adam, Y.; Lin, J.; Wei, D. Biochemical and structural analysis of Gox2181, a new member of the SDR superfamily from Gluconobacter oxydans. Biochem Biophys Res. Commun., 2011, 415(2), 410-415.

52. Haft, D. H.; Pierce, P. G.; Mayclin, S. J.; Sullivan, A.; Gardberg, A. S.; Abendroth, J.; Begley, D. W.; Phan, I. Q.; Staker, B. L.; Myler, P. J.; Marathias, V. M.; Lorimer, D. D.; Edwards, T. E. Mycofactocin-associated mycobacterial dehydrogenases with non-exchangeable NAD cofactors. Sci Rep., 2017, 25, 7:41074.

53. Zaccai, N. R.; Carter, L. G.; Berrow, N. S.; Sainsbury, S.; Nettleship, J. E.; Walter, T. S.; Harlos, K.; Owens, R. J.; Wilson, K. S.; Stuart, D. I.; Esnouf, R. M. Crystal structure of a 3-oxoacyl-(acylcarrier protein) reductase (BA3989) from Bacillus anthracis at 2.4-A resolution. Proteins, 2008, 70(2), 562-567.

54. Nishioka, T.; Yasutake, Y.; Nishiya, Y.; Tamura, T. Structure-guided mutagenesis for the improvement of substrate specificity of Bacillus megaterium glucose 1dehydrogenase IV. FEBS J., 2012, 279(17), 3264-3275.

55. Haft, D. H.; Pierce, P. G.; Mayclin, S. J.; Sullivan, A.; Gardberg, A. S.; Abendroth, J.; Begley, D. W.; Phan, I. Q.; Staker, B. L.; Myler, P. J.; Marathias, V. M.; Lorimer, D. D.; Edwards, T. E. Mycofactocin-associated mycobacterial dehydrogenases with nonexchangeable NAD cofactors. Sci Rep., 2017, 25(7), 41074.

56. Yamashita, A.; Endo, M.; Higashi, T.; Nakatsu, T.; Yamada, Y.; Oda, J.; Kato, H. Capturing enzyme structure prior to reaction initiation: tropinone reductase-II-substrate complexes. Biochemistry, 2003, 42(19), 5566-5573.

57. Carius, Y.; Christian, H.; Faust, A.; Zander, U.; Klink, B. U.; Kornberger, P.; Kohring, G. W.; Giffhorn, F.; Scheidig, A. J. Structural insight into substrate differentiation of the sugar-metabolizing enzyme galactitol dehydrogenase from Rhodobacter sphaeroides D. J Biol. Chem., 2010, 285(26), 20006-20014.

58. Baugh, L.; Phan, I.; Begley, D. W.; Clifton, M. C.; Armour, B.; Dranow, D. M.; Taylor, B. M.; Muruthi, M. M.; Abendroth, J.; Fairman, J. W.; Fox, D.; Dieterich, S. 
H.; Staker, B. L.; Gardberg, A. S.; Choi, R.; Hewitt, S. N.; Napuli, A. J.; Myers, J.; Barrett, L. K.; Zhang, Y.; Ferrell, M.; Mundt, E.; Thompkins, K.; Tran, N.; LyonsAbbott, S.; Abramov, A.; Sekar, A.; Serbzhinskiy, D.; Lorimer, D.; Buchko, G. W.; Stacy, R.; Stewart, L. J.; Edwards, T. E.; Van Voorhis, W. C.; Myler, P. J. Increasing the structural coverage of tuberculosis drug targets. Tuberculosis (Edinb), 2015, 95(2), 142-148.

59. Javidpour, P.; Pereira, J. H.; Goh, E. B.; McAndrew, R. P.; Ma, S. M.; Friedland, G. D.; Keasling, J. D.; Chhabra, S. R.; Adams, P. D.; Beller, H. R. Biochemical and structural studies of NADH-dependent FabG used to increase the bacterial production of fatty acids under anaerobic conditions. Appl Environ Microbiol., 2014, 80(2), 497505. 


\section{Figures legends}

Fig. 1. A, MtbDprE2 sequence showing the residues involved in NADH binding (blue) and in substrate-binding (red). B, Restriction-digestion analysis of SUMO-MtbDprE2 plasmid showing the MtbDprE2 gene fall out. C, SDS-PAGE analysis of SUMO-MtbDprE2 fusion protein after cleavage with SUMO protease (1- SUMO and 2-MtbDprE2). The cleaved MtbDprE2 eluted as oligomer from Superdex 200(16/60) column, as identified using molecular mass standard.

Figure 2. Secondary structure and thermal denaturation analysis of $M t b D p r E 2$ using CD spectroscopy. (A) The PSIPRED analysis showing the secondary structural contents of MtbDprE2. The $\alpha$-helices are shown magenta spiral and $\beta$-strands in yellow arrow. (B) The 260 to $200 \mathrm{~nm}$ wavelength was used for CD data collection on MtbDprE2 and secondary structures were estimated by Dichroweb server [41]. Inset shows the MtbDprE2 secondary structure (C) The CD data collected $260-200 \mathrm{~nm}$ range starting from $20{ }^{\circ} \mathrm{C}$ to $70{ }^{\circ} \mathrm{C}$ range with $10{ }^{0} \mathrm{C}$ interval.

Figure 3. (A) The MtbDprE2 model (1-254 residues) obtained from I-TASSER server [37]. The $\alpha$-helices in cyan, $\beta$-sheets in magenta and loops are shown in light orange colors respectively. (B) Electrostatic surface diagram of MtbDprE2 contoured at $-10 \mathrm{kT}$ to $+10 \mathrm{kT}$. Overall surface of MtbDprE2 is negatively charged, while positive charges spread around the surface. The NADH cofactor binding site is partially positively charged. (C) The LigPlot [52] showing the interactions between NADH and MtbDprE2. Hydrogen bonds are shown in green dashed lines and van der waals interactions in orange Arc color (D) Stereogram of 
MtbDprE2+NADH complex model. The NADH is shown in green color. (E) Electrostatic surface diagram of MtbDprE2 contoured at -10kT to +10kT with NADH fitted in the binding pocket.

Figure 4. (A) Sequence alignment of MtbDprE2 sequence with ten closest structural homologs obtained from PDB database, (i) PDB-3awd (red) (ii) PDB-3s55 (yellow) (iii) PDB-1gee (blue) (iv) PDB-2uvd (magenta) (v) PDB-3aut (orange) (vi) PDB-3t7c (wheat) (vii) PDB-1ipe (grey) (viii) PDB-2wsb (green) (ix) PDB=2uve (light pink) (x) PDB-4nbv (light blue) using MultiAln and ESPript programs. The secondary structures of MtbDprE2 model are shown above sequence alignment. The highly conserved residues are shown in red shade and semi conserved in red letters. The NADH binding residues are indicated in (*) above sequence alignment. (B) Superposition of (i) PDB-3awd (red) (ii) PDB-3s55 (yellow) (iii) PDB-1gee (blue) (iv) PDB-2uvd (magenta) (v) PDB-3aut (orange) (vi) PDB3t7c (wheat) (vii) PDB-1ipe (grey) (viii) PDB-2wsb (green) (ix) PDB=2uve (light pink) (x) PDB-4nbv (light blue) on MtbDprE2 structure (cyan) using PyMol program [22]. The small domain of MtbDprE2 having $\alpha 6$ and $\alpha 7$ helices showed large conformational changes (shown in circle) and minor conformational changes were observed in major domain. Residues involved in NADH binding are shown in stick diagram.

Fig. 5. Molecular dynamics simulation on MtbDprE2 and its complex with NADH (Table 3). A, Simulated MtbDprE2 model (red) is superposed initial MtbDprE2 model (grey). B, Simulated MtbDprE2+NADH model (cyan) is superposed on initial MtbDprE2+NADH model (grey). C, Conformational changes in NADH binding residues after dynamics 
simulation, initial (red) and simulated (cyan). D, RMSD of the backbone Ca atoms (in nm) obtained 1 ns simulation of apo and complexed MtbDprE2. E, Radius of gyration (in nm) of apo and complexed MtbDprE2 during 1 ns simulation. F, The root square fluctuation (RMSF in $\AA$ ) of backbone C $\alpha$ atoms of apo and complexed MtbDprE2 during $1 \mathrm{~ns}$ simulation.

\section{Supplementary figures}

Figure S1. Quality check on apo-MtbDprE2 model obtained after 1 ns of dynamics simulation. (A) The 95\% residues in most favored and 5\% residues in additionally allowed regions were obtained in the Ramachandran plot. (B) Z score of -5.5 was observed in ProSA plot, which indicate that overall quality of model is good. (C) the regions (yellow color) in model, that can be rejected with 95\% confidence is shown in ERRAT plot. (D) the regions, whose threshold scores greater than 0.2 is shown in Verify 3D plot.

Figure S2. Quality check on $M t b D p r E 2+\mathrm{NADH}$ complex obtained after $1 \mathrm{~ns}$ of dynamics simulation. (A) 96\% residues in most favored and 4\% residues in additional allowed regions were observed in Ramachandran plot (B) Z score of - 7.36 was observed in ProSA plot, which indicate that model has good quality. (C) ERRAT plot shows the regions, that can be rejected with $95 \%$ confidence (yellow). (D) the regions whose threshold scores greater than 0.2 is shown in Verify 3D plot. 
Table 1. Secondary structural composition of MtbDprE2 obtained from circular dichroism and theoretical structure prediction programs

\begin{tabular}{|l|l|l|l|}
\hline Programs & $\boldsymbol{\alpha}$-helix & $\boldsymbol{\beta}$-sheet & Random coil \\
\hline CD & $\mathbf{4 7 . 6 \%}$ & $\mathbf{1 9 . 8 \%}$ & $\mathbf{3 2 . 6 \%}$ \\
\hline SPOMA18 & $46.5 \%$ & $25.2 \%$ & $28.3 \%$ \\
\hline GOR4 & $54.3 \%$ & $11.4 \%$ & $34.2 \%$ \\
\hline PHD & $46.5 \%$ & $16.1 \%$ & $37.4 \%$ \\
\hline DSC & $40.5 \%$ & $15.7 \%$ & $43.7 \%$ \\
\hline HNN & $53.5 \%$ & $15.7 \%$ & $30.7 \%$ \\
\hline RAPTOR & $44 \%$ & $12 \%$ & $42 \%$ \\
\hline
\end{tabular}


Table 2. Top 10 best structural analogs of MtbDprE2 from PDB

\begin{tabular}{llllll}
\hline Rank & PDB hits & TM-score & RMSD $^{\mathrm{b}}$ & IDEN $^{\mathrm{c}}$ & Cov $^{\mathrm{d}}$ \\
\hline 1 & 3awdA & 0.874 & 2.62 & 0.174 & 0.972 \\
2 & 3s55A & 0.874 & 2.47 & 0.184 & 0.965 \\
3 & 1geeA & 0.873 & 2.49 & 0.154 & 0.969 \\
4 & 2uvdH & 0.873 & 2.54 & 0.156 & 0.957 \\
5 & 3autA & 0.872 & 2.60 & 0.166 & 0.972 \\
6 & 3t7cA & 0.871 & 2.58 & 0.133 & 0.972 \\
7 & 1ipeA & 0.870 & 2.58 & 0.181 & 0.976 \\
8 & 2wsbA & 0.870 & 2.57 & 0.184 & 0.965 \\
9 & 3uveA & 0.869 & 2.62 & 0.150 & 0.972 \\
10 & 4 nbvA & 0.869 & 2.51 & 0.210 & 0.957
\end{tabular}
(a) TM-score- Ranking of protein
(b) RMSD- RMSD between structural analogs and MtbDprE2 residues
(c) IDEN- sequence identity
(d) Cov- coverage of the alignment by TM-align 
Table 3. Dynamics simulation on apo-MtbDrpE2 and its complex with NADH

\begin{tabular}{lll}
\hline Models & Apo & NADH \\
\hline Protein atoms & 2452 & 2444 \\
Water/ion atoms & $23098 / 17 \mathrm{Na} / 18 \mathrm{Cl}$ & $32,276 / 23 \mathrm{Na} / 22 \mathrm{Cl}$ \\
Ligand atoms & - & 47 \\
Total atoms & 26,586 & 34,824 \\
NVT equilibration & $2.5 \mathrm{~ns}$ & $2.5 \mathrm{~ns}$ \\
NPT equilibration & $2.5 \mathrm{~ns}$ & $2.5 \mathrm{~ns}$ \\
Time steps (fs) & 2 & 2 \\
Simulation (ns) & 1 & 1
\end{tabular}


bioRxiv preprint doi: https://doi.org/10.1101/2020.10.15.341941; this version posted October 16,2020 . The copyright holder for this preprint (which was not certified by peer review) is the author/funder. All rights reserved. No reuse allowed without permission.

A

1 MVLDAVGNPQTVLLLGGTSEIGLAICERYLHNSAARIVLACLPDDPRRED 50

51 AAAAMKQAGARSVELIDFDALDTDSHPKMIEAAFSGGDVDVAIVAFGLLG 100

101 DAEELWQNQR KAVQIAEINYTAAVSVGVLLAEKMRAQGFGQIIAMSSAAG 150

151 ERVRRANFVYGSTKAGLDGF YLGLSEALREYGVRVLVIRPGQVRTRMSAH 200

201 LKEAPLTVDKEYVANLAVTA SAKGKELVWA PAAFRYVMMVLRHIPRSIFR 250

251 KLPI

B

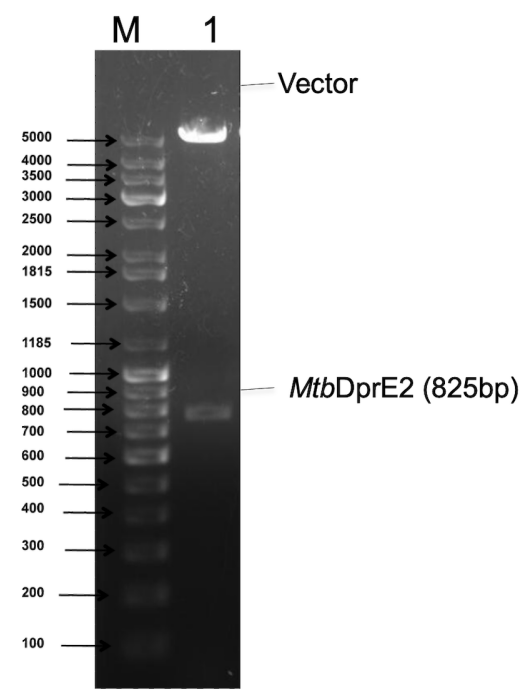

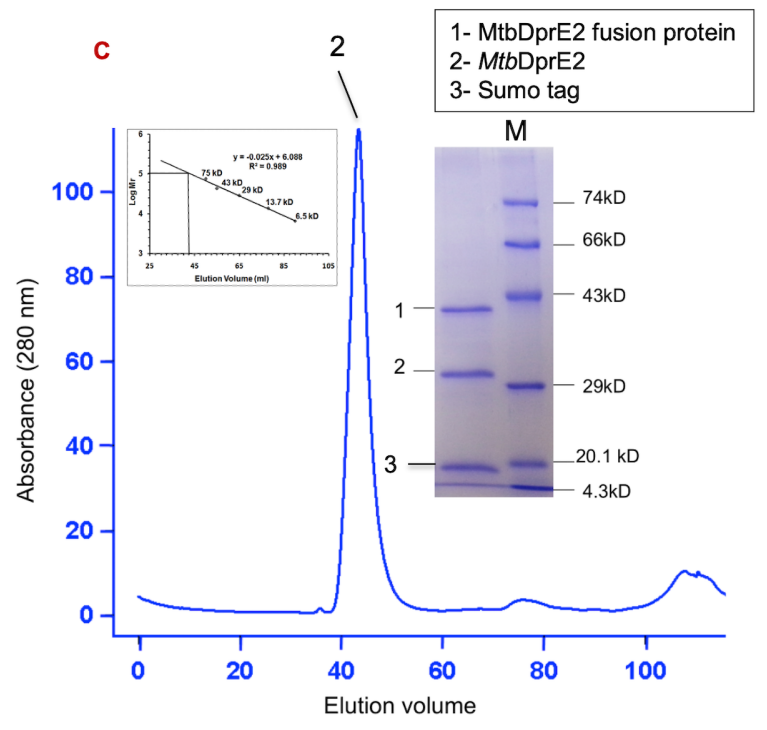


bioRxiv preprint doi: https://doi.org/10.1101/2020.10.15.341941; this version posted October 16,2020 . The copyright holder for this preprint (which was not certified by peer review) is the author/funder. All rights reserved. No reuse allowed without permission.
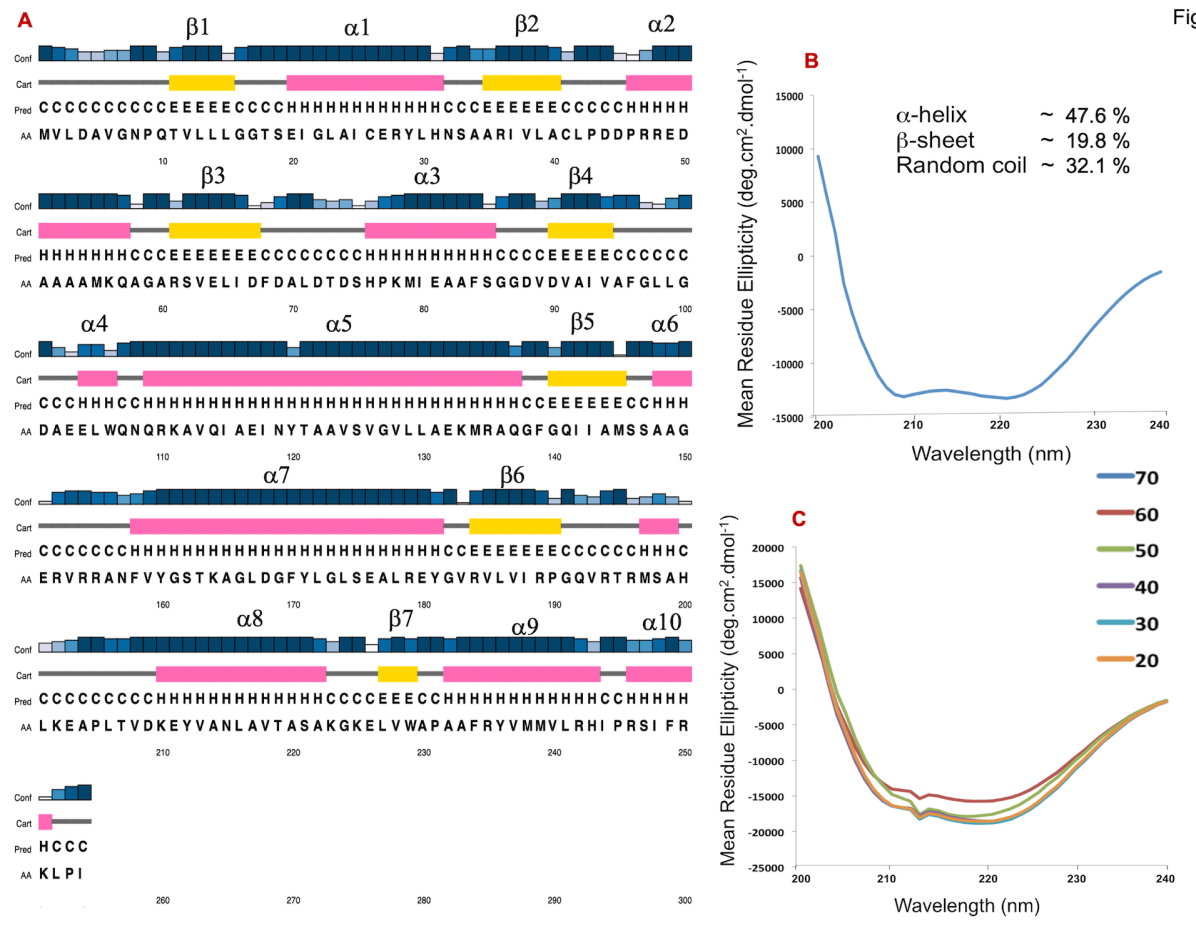
bioRxiv preprint doi: https://doi.org/10.1101/2020.10.15.341941; this version posted October 16,2020 . The copyright holder for this preprint (which was not certified by peer review) is the author/funder. All rights reserved. No reuse allowed without permission.

A

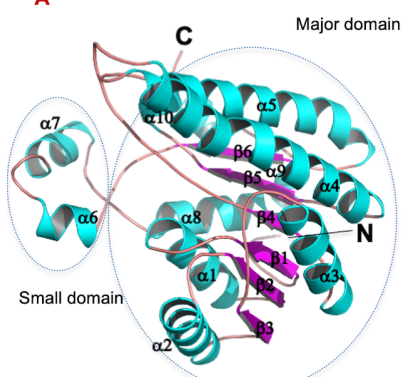

D

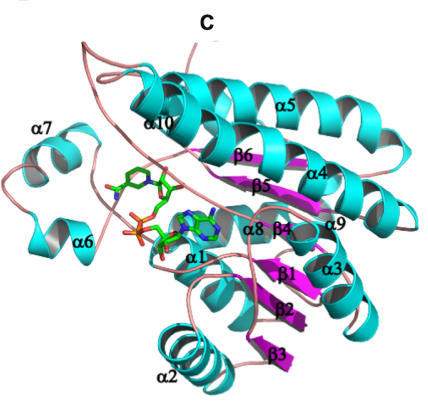

B

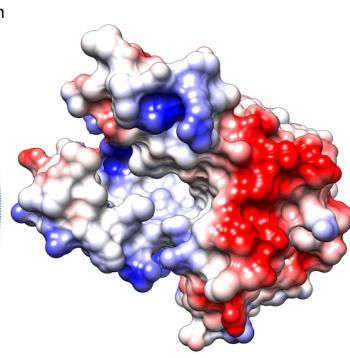

E

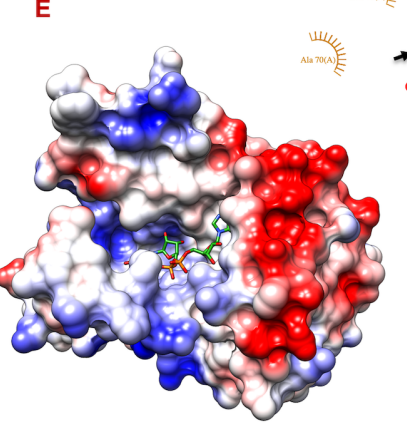

Fig. 3

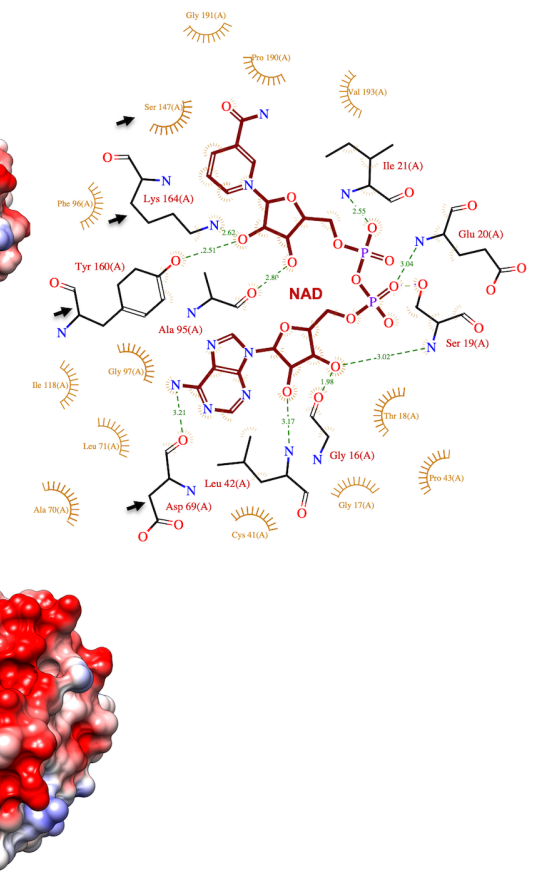


bioRxiv preprint doi: https://doi.org/10.1101/2020.10.15.341941; this version posted October 16, 2020. The copyright holder for this preprint (which was not certified by peer review) is the author/funder. All rights reserved. No reuse allowed without permission.

A Mtbop
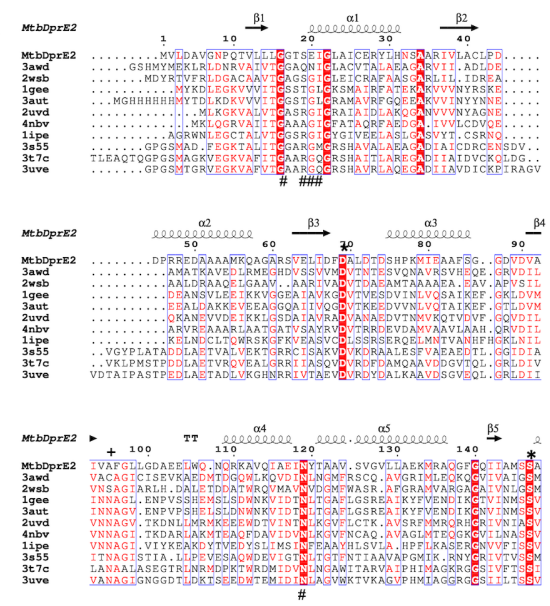
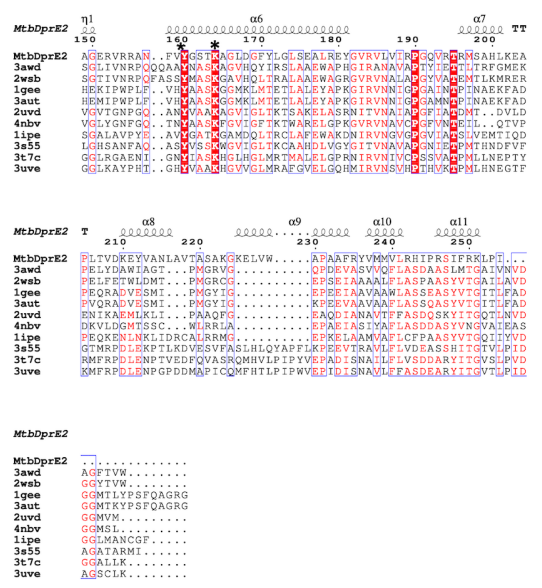

B

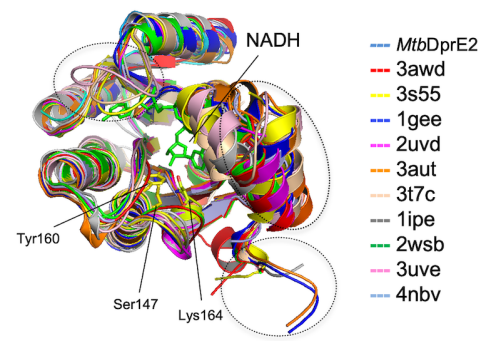

Fig. 4 
bioRxiv preprint doi: https://doi.org/10.1101/2020.10.15.341941; this version posted October 16,2020 . The copyright holder for this preprint (which was not certified by peer review) is the author/funder. All rights reserved. No reuse allowed without permission.

A

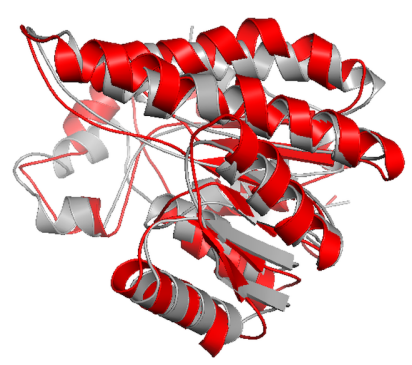

B

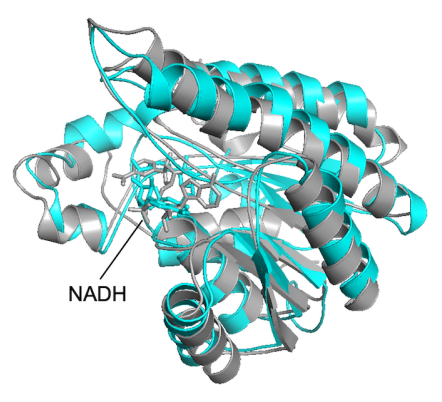

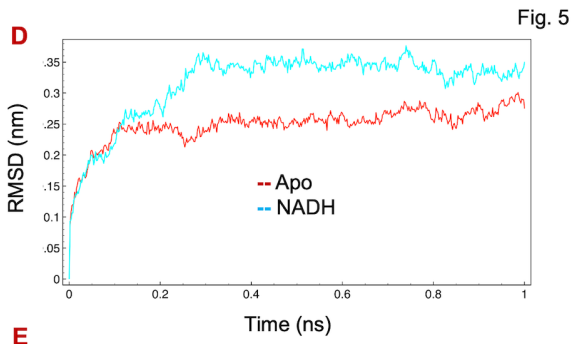

E
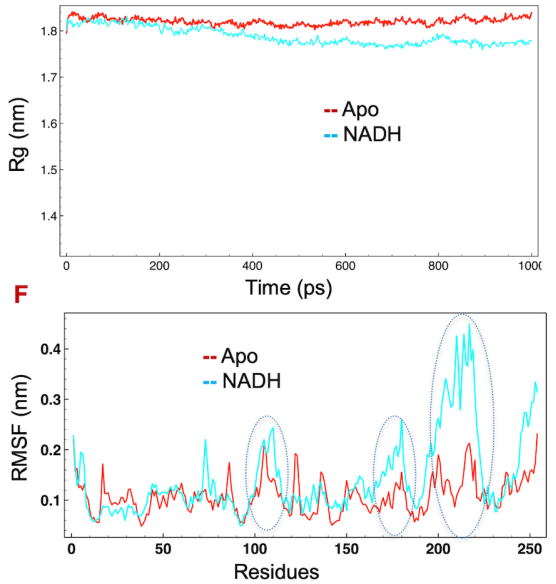
bioRxiv preprint doi: https://doi.org/10.1101/2020.10.15.341941; this version posted October 16, 2020. The copyright holder for this preprint (which was not certified by peer review) is the author/funder. All rights reserved. No reuse allowed without permission. 
bioRxiv preprint doi: https://doi.org/10.1101/2020.10.15.341941; this version posted October 16,2020 . The copyright holder for this preprint (which was not certified by peer review) is the author/funder. All rights reserved. No reuse allowed without permission.

A

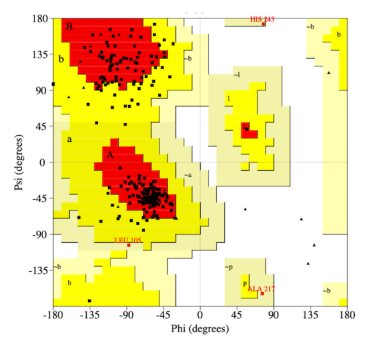

B

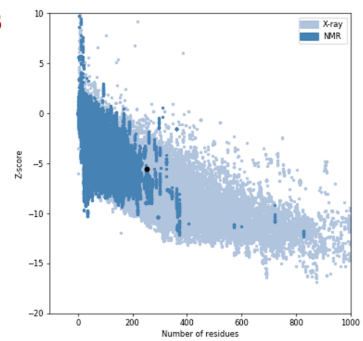

Fig. S1

Averaged Score Raw Score

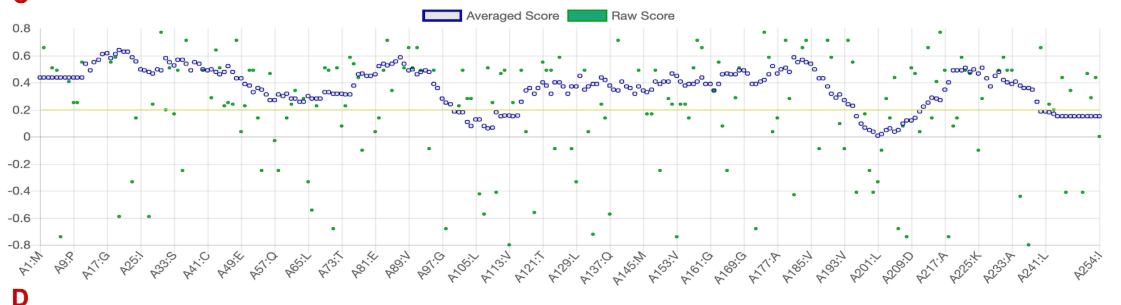

.

ERRAT Error Values

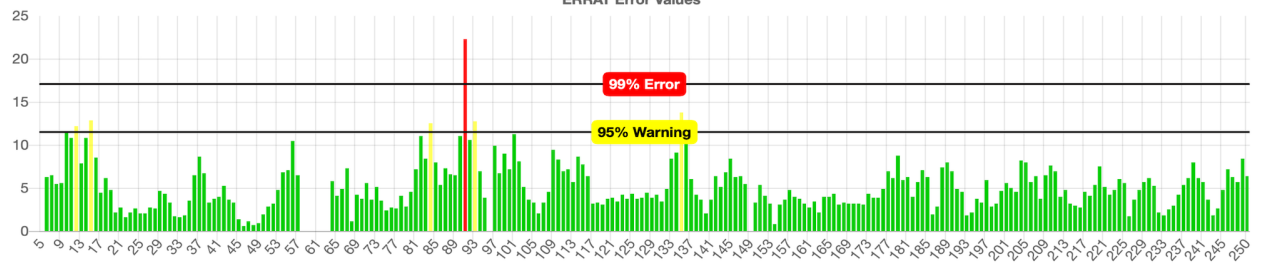


bioRxiv preprint doi: https://doi.org/10.1101/2020.10.15.341941; this version posted October 16, 2020. The copyright holder for this preprint (which was not certified by peer review) is the author/funder. All rights reserved. No reuse allowed without permission.

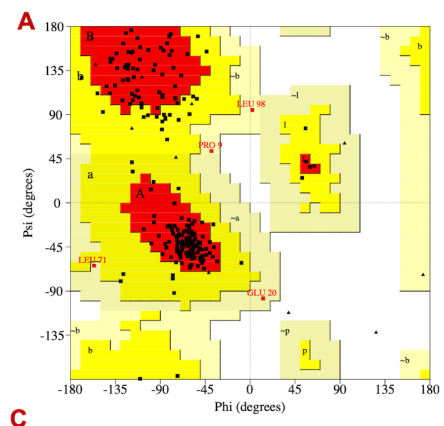

C

B

Fig. S2

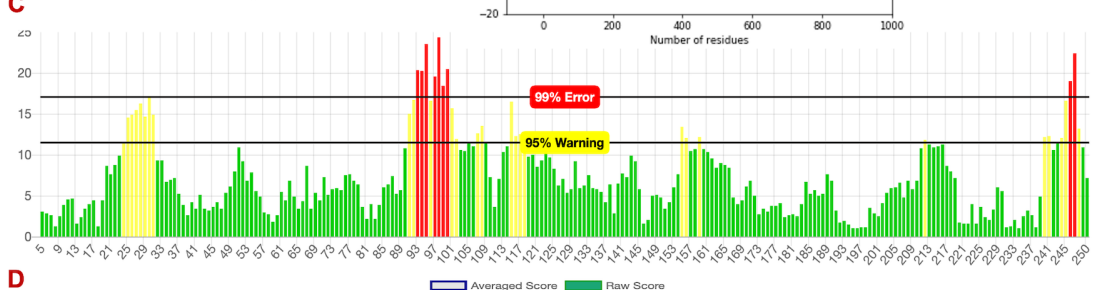

D

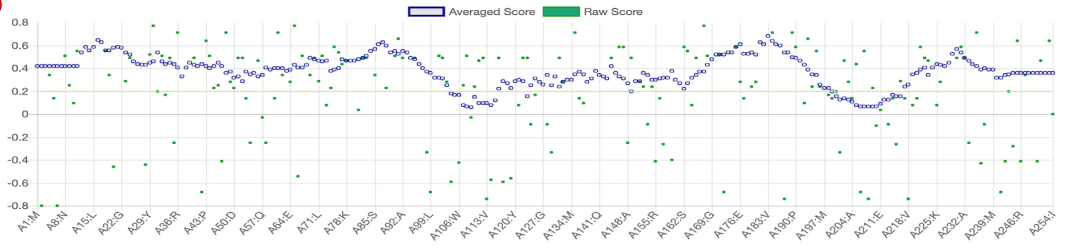

